\title{
Correction: In vitro expansion impaired the stemness of early passage mesenchymal stem cells for treatment of cartilage defects
}

Tongmeng Jiang ${ }^{1,2}$, Guojie Xu ${ }^{1,2}$, Qiuyan Wang ${ }^{3}$, Lihui Yang ${ }^{4}$, Li Zheng ${ }^{1,5}$, Jinmin Zhao ${ }^{1,2,5,6}$ and Xingdong Zhang ${ }^{7}$

\section{Correction to: Cell Death and Disease https://doi.org/10.1038/cddis.2017.215 published online 01 June 2017}

Following the publication of this article, the authors noticed that Fig. $5 \mathrm{c}$ in the original paper is incorrect due to an error in image handling during the creation of the figure. Specifically, the image for the group P3 BMSCs of time point $21 \mathrm{~d}$ and the image in the group BMMNCs of time point $14 \mathrm{~d}$ were incorrect. These linked to the group for BMMNCs of time point $21 \mathrm{~d}$, which was also, as a result, incorrect. The correct figure is shown below. The error does not impact the conclusions of the article. The authors would like to apologize for any inconvenience this may have caused.

Published online: 26 September 2019

Correspondence: Li Zheng (zhengli224@163.com) or Jinmin Zhao (zhaojinmin@126.com)

${ }^{1}$ Guangxi Engineering Center in Biomedical Materials for Tissue and Organ Regeneration, The First Affiliated Hospital of Guangxi Medical University, Nanning 530021, China

${ }^{2}$ Department of Orthopaedics Trauma and Hand Surgery, The First Affiliated Hospital of Guangxi Medical University, Nanning 530021, China

${ }^{3}$ Center for Genomic and Personalized Medicine, Guangxi Medical University, Nanning 530021, China

${ }^{4}$ School of Nursing, Guangxi Medical University, Nanning 530021, China

${ }^{5}$ Collaborative Innovation Center of Guangxi Biological Medicine, The First Affiliated Hospital of Guangxi Medical University, Nanning 530021, China

${ }^{6}$ Guangxi Key Laboratory of Regenerative Medicine, The First Affiliated Hospital of Guangxi Medical University, Nanning 530021, China

${ }^{7}$ National Engineering Research Center for Biomaterials, Sichuan University, Chengdu 610064, China

These authors contributed equally: Tongmeng Jiang, Guojie Xu, Qiuyan Wang 


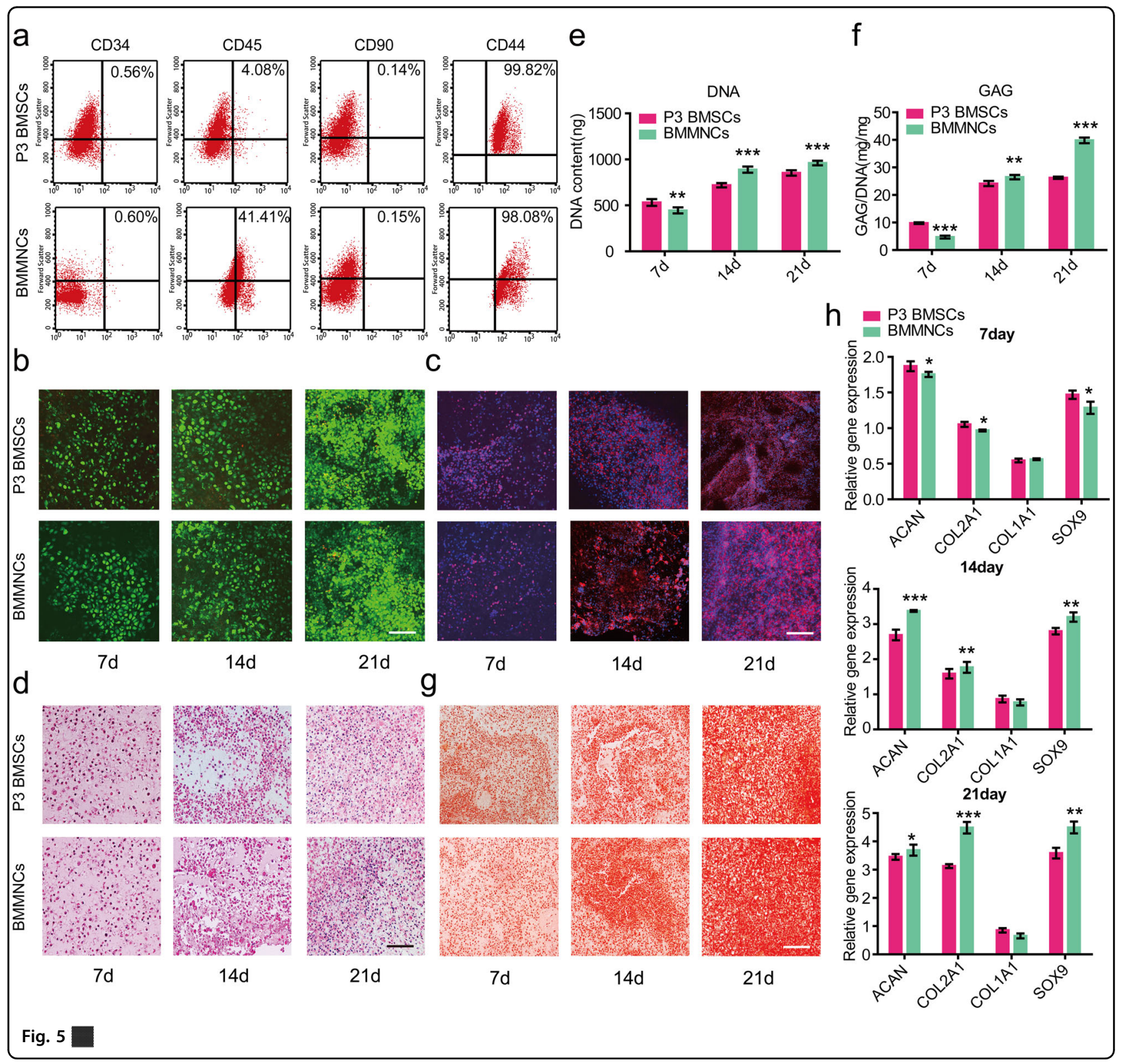

